\title{
Análisis de un método activo para la detección de isla basado en la perturbación de la fase en el pll y comparación con otros métodos activos
}

\author{
Cesar L. Trujillo Rodríguez * \\ David Velasco de la Fuente $* *$ \\ Emilio Figueres Amorós $* * *$ \\ Gabriel Garcerá Sanfeliú $* * * *$
}

\section{Resumen}

En este artículo se propone un método nuevo para la detección de isla en sistemas de generación distribuida. Dicho procedimiento se basa en la introducción de una perturbación a la salida del inversor con el fin de observar el comportamiento de la tensión en el punto de acople común (PCC), la cual dependerá de la impedancia conectada —al PCC — en la situación de isla. Así mismo, se efectuó un análisis comparativo entre las principales técnicas activas residentes en el inversor para detección de isla y su similitud con la propuesta. Los algoritmos mencionados en este artículo fueron simulados en PSIM ${ }^{\mathrm{TM}}$ y comparados para establecer sus ventajas y desventajas (basados en estándares IEEE).

\section{Palabras clave}

Inversores, técnicas activas de detección de isla, generadores distribuidos.

\section{ANALYSIS OF AN ACTIVE ANTI-ISLANDING METHOD BASED ON PHASE-PLL PERTURBATION AND COMPARISON WITH OTHERS ACTIVE METHODS}

\begin{abstract}
This paper presents a new active anti-islanding detection method for distributed power generation systems. This method is based on introducing a disturbance at the inverter output and observing the behavior of the voltage at the point of common coupling (PCC), which depends on the impedance connected to the PCC in an islanding situation. Additionally, this paper presents the analysis and comparison of the main active techniques
\end{abstract}

* Profesor de la Facultad de Ingeniería de la Universidad Distrital Francisco José de Caldas. cltrujillo@udistrital.edu.co

** Candidato a Doctor de la Universidad Politécnica de Valencia. davede@posgrado.upv.es,

*** Profesor Departamento de Ingeniería Electrónica, Universidad Politécnica de Valencia. efiguere@eln.upv.es

**** Profesor Departamento de Ingeniería Electrónica, Universidad Politécnica de Valencia. ggarcera@eln.upv.es 
for islanding detection and these were compare with the proposed technique. The algorithms mentioned in this work were simulated in PSIM $^{\mathrm{TM}}$ in order to make a comparative analysis and to establish their advantages and disadvantages according to IEEE standards.

\section{Key words}

Inverters, active islanding detection methods, distributed generators.

\section{INTRODUCCIÓN}

La condición de isla (islanding) en sistemas de potencia distribuidos es un fenómeno que se presenta cuando la energía suministrada por la red eléctrica es interrumpida debido a varios factores y los generadores distribuidos (DGs) continúan energizando una parte o la totalidad de la carga. Entonces, la red eléctrica deja de controlar esa fracción aislada del sistema de distribución — contiene tanto carga como generación-, comprometiendo la seguridad, el restablecimiento del servicio y la fiabilidad del equipo (IEAPVPS T5-09, 2002).

La idea principal para detectar la condición de islanding es supervisar los parámetros de salida de los DGs y/o del sistema; así se determina si se han presentado cambios que posibiliten o no la existencia de dicha condición. Las técnicas de detección de islanding se dividen en técnicas remotas y técnicas locales y éstas, a su vez, se componen de pasivas y activas - ver figura 1. (Mahat, P., Chen, Z. y BakJensen, B.; 2008).

Es importante resaltar dos características fundamentales para entender el fenómeno de islanding. La primera, asociada a la denominada "zona de nodetección" (NDZ), se puede definir como el rango (en términos de la diferencia de potencia entre el inversor del DG y la carga o los parámetros de carga) en el cual el esquema de detección de islanding - bajo prueba - falla para detectar dicha condición (De Mango et ál., 2006). Por su parte, la segunda hace referencia al tipo de cargas (potenciales dentro de la isla) que pueden ser modeladas como un circuito RLC paralelo. La razón de utilizar dicho modelo de carga se debe, fundamentalmente, a que son las que generan mayor grado de dificultad para la mayoría de técnicas de detección —en el momento de identificar si existe una condición de islanding. En general, las cargas no lineales, como las que producen corrientes armónicas o potencia constante, no presentan tanta dificultad en la detección de islanding (IEAPVPS T5-09, 2002). Entre tanto, las técnicas activas residentes en el inversor introducen perturbaciones a la salida del mismo con el fin de afectar un parámetro y así detectar la condición de islanding.

\section{FIGURA 1. Clasificación de técnicas anti-islanding.}

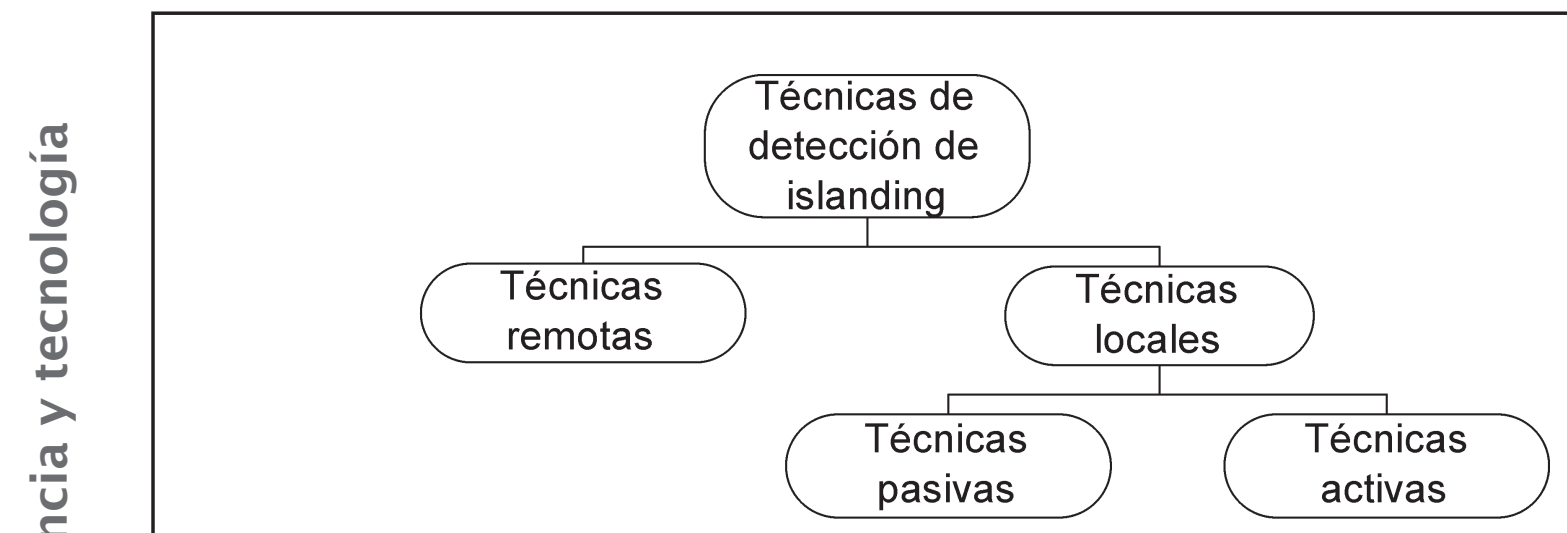


El primer segmento de este artículo describe la composición el sistema DG conectado a la red eléctrica. Entre tanto, los diversos métodos activos residentes en el inversor, así como el método propuesto, son descritos en el segundo segmento $y$, para finalizar, el contenido de la última parte del escrito está constituido por la simulación de dichos métodos y el análisis de los resultados con carga RLC según estándares.

\section{DESCRIPCIÓN DEL INVERSOR}

En la figura 1 se presenta el esquema del inversor implementado. Dicho inversor se alimenta de un arreglo de dos paneles fotovoltaicos, de 220 W cada uno, organizados paralelamente; esto hace necesario una etapa DC-DC que eleve la tensión suministrada por los paneles a niveles adecuados para que el sistema pueda interconectarse con la red eléctrica. La etapa utilizada para dicha acción fue un push-pull, mientras que para la conversión DC-AC se empleó un inversor a PWM en puente $\mathrm{H}$ con un filtro LCL a la salida (Liserre, M. et ál., 2006), (Liang, J. et ál., 2003).

En cuanto al inversor, la corriente de referencia del controlador de corriente se calcula teniendo en cuenta la cantidad de potencia activa y reactiva capaz de ser entregada por el DG. Así mismo, dicha corriente se sincroniza con la componente fundamental de la tensión de red a través de un phase lockeed loop (dqPLL) implementado mediante la técnica del marco de referencia síncrono (Ciobotaru, M.; Teodorescu, R. y Blaabjerg, F., 2006), (Liserre, M. et ál, 2005). El esquema del dqPLL se muestra en la figura 3.

Los principales bloques constitutivos de control utilizados en éste sistema son: el seguidor del punto de máxima potencia (MPPT), desarrollado a través del algoritmo $\mathrm{P} \& \mathrm{O}$ (Esram, T. y Chapman, P.; 2007); el control del DC-Link, implementado a través de un controlador PI; y el controlador de corriente, el cual se implementó con un compensador armónico (Kazmierkowski, M.; Krishnan, R., y Blaabjerg, F., 2002) con el fin de cumplir lo estable-
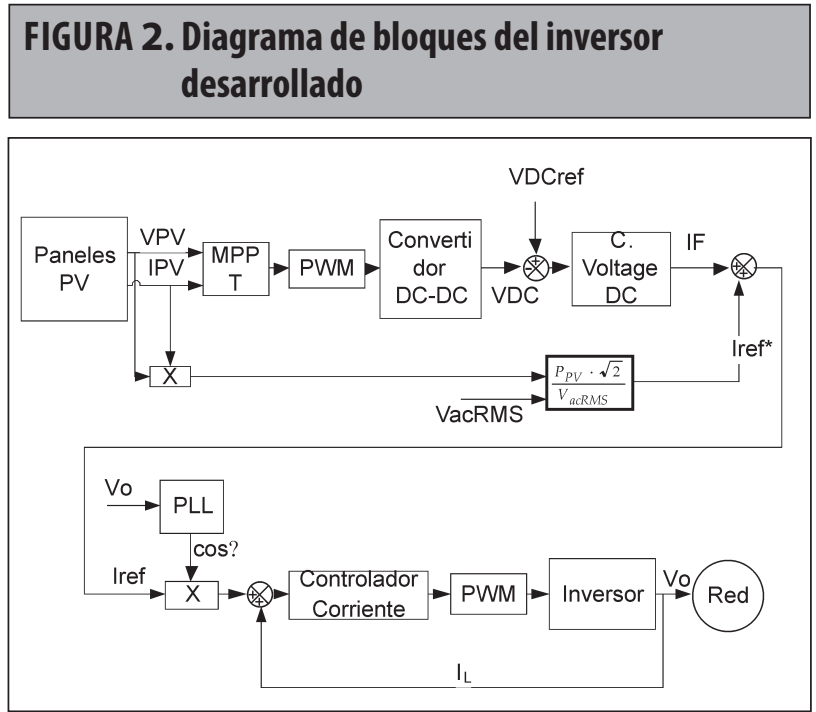

FIGURA 3. Esquema del dqPLL.

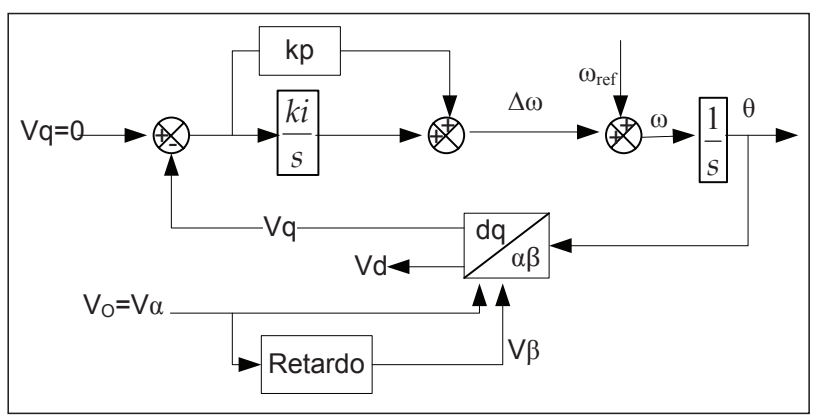

cido por el estándar IEEE 929-2000 (IEEE Std. 929; 2000), en términos del THD de corriente.

\section{MÉTODOS ACTIVOS DE DETECCIÓN DE ISLA}

Las técnicas activas introducen intencionalmente perturbaciones a la salida del inversor con el fin de determinar si se afectan los parámetros de tensión, frecuencia e impedancia (de ser éste el caso se asume que la red se ha desconectado y se aísla el inversor de la carga). Además, tienen la ventaja de reducir notablemente — algunas la eliminan-la NDZ; sin embargo, para logar tal propósito deterioran en mayor o menor grado la calidad de potencia.

Los métodos activos pueden ser clasificados en: basados en realimentación positiva y basados en inyección de armónicos. 


\section{Métodos activos basados en realimentación positiva}

Presentan la particularidad de afectar controladamente, de forma individual o combinada, la tensión, la frecuencia y demás parámetros asociados al inversor. Entre tanto, cuando se presenta un cambio que aparta de un umbral preasignado a dichas variables; entonces, a través de la realimentación positiva de la variable - si la red no se encuentra conectada - se alcanzan los niveles de sobre/subtensión y sobre/sub-frecuencia suficientes para detectar la situación de islanding.

Existen diferentes métodos activos, basados en realimentación positiva, reportados en la literatura. Algunos de ellos son: El método de variación de la potencia activa y de la potencia reactiva (De Mango, F. et ál; 2006), (Jeraputra, C. y Enjeti, P.; 2004) y los Esquemas de frecuencia de General Electric (Gefs), (Huili, S.; Lopes, L. y Zhixiang, L.; 2004), (Ye, Z. et ál. 2004); entre otros.

\section{Variación de la potencia activa y de la poten- cia reactiva}

En condición de islanding la potencia fluye desde el inversor hacia la carga, afectando tanto a la corriente como a la tensión en el PCC. La alteración de la tensión frente a la variación de la potencia activa, inyectada por el inversor a la carga durante la condición de islanding, se define como:

$$
\mathrm{P}_{\mathrm{DG}}=\mathrm{P}_{\mathrm{LOAD}}=\frac{\mathrm{V}^{2}}{\mathrm{R}}
$$

Derivando $P_{D G}$ con respecto a la tensión y expresando ésta en términos de la potencia se obtiene:

$$
\frac{\partial P_{D G}}{\partial V}=2 \cdot \frac{V}{R}=2 \cdot \sqrt{\frac{P_{D G}}{R}}
$$

Entonces, la variación de la tensión, en términos de la potencia, se puede expresar como:

$$
\Delta V=\frac{\Delta P_{D G}}{2} \cdot \sqrt{\frac{R}{P_{D G}}}
$$

Como $\mathrm{R}_{\text {y }} \mathrm{P}_{\mathrm{DG}}$ son constantes en el punto de operación en régimen permanente, la variación de la tensión es directamente proporcional a la variación de la potencia activa. Por lo tanto, es posible modificar la potencia activa inyectada por el inversor con el fin de llevar la amplitud de la tensión fuera del rango de operación normal y así detectar el islanding.

Es necesario escoger cuidadosamente el momento de inyección de la potencia, pues las variaciones continúas de la potencia inyectada pueden perturbar el seguimiento del punto de máxima potencia (MPPT). Por estas razones, el método se basa en la inyección de potencia activa únicamente cuando la tensión medida en el PCC supere un valor umbral determinado (Vs).

El tiempo necesario para que el algoritmo detecte una falla puede ajustarse a través de un factor $\mathrm{K}_{\mathrm{v}}$ que aumente o disminuya el término $\mathrm{dP}$ de forma proporcional a la variación de la tensión. Éste valor debe ser lo suficientemente grande para provocar la detección de islanding sin agenerar sobrecorrientes que afecten los elementos del sistema. Un valor inicial de la constante se puede conseguir a partir de (3). La referencia de corriente en el control del inversor puede ser calculada de la siguiente manera:

$$
\mathrm{I}_{\text {ref }}=\frac{d P+P_{D G}}{V}
$$

Donde $\mathrm{dP}=\mathrm{K}_{\mathrm{v}}\left(\mathrm{V}-\mathrm{V}_{\mathrm{n}}\right)$, siendo $\mathrm{V}_{\mathrm{n}}$ la amplitud de la tensión nominal y $\mathrm{V}$ la medición de la amplitud de la tensión realimentada.

De manera similar a la relación entre tensión y potencia activa, existe una fuerte dependencia entre frecuencia y potencia reactiva, hecho que permite desarrollar otro método de detección de islanding basado en la medición de la frecuencia de red. Debido a que el estándar IEEE 929-2000 recomienda la operación de los DGs cercana a un factor de potencia unitario, la potencia reactiva generada debe ser igual a cero. Durante el comienzo de la situación de islanding, la ecuación (5) sigue siendo válida, por lo que la desviación de la frecuencia dependerá de los valores de las componentes inductivas y 
capacitivas de la carga. Entonces, la variación de Q con respecto a $\omega$ se presenta en en (6), donde $\omega_{0}$ es la frecuencia de resonancia del circuito equivalente paralelo RLC —ecuación (7)-:

$$
\begin{gathered}
\mathrm{Q}_{\mathrm{DG}}=\mathrm{Q}_{\mathrm{LOAD}}=0=\mathrm{V}^{2}\left(\frac{1}{\omega \mathrm{L}}-\omega \mathrm{C}\right) \\
\frac{Q-0}{\omega-\omega_{O}}=\left.\frac{d Q}{d \omega}\right|_{\omega=\omega_{O}} \\
\omega_{\mathrm{O}}=\frac{1}{\sqrt{\mathrm{LC}}}
\end{gathered}
$$

Resolviendo (7), con el fin de determinar la variación de la frecuencia como una función de $\mathrm{Q}$ y fijando la frecuencia de la carga igual a la frecuencia de resonancia, se obtiene:

$$
\Delta \omega=-V^{2} \cdot\left[\frac{\omega_{0}^{2} \mathrm{~L}}{\omega_{0}^{2} \mathrm{LC}+1}\right] \cdot \mathrm{Q}
$$

Como se observa en (8), la variación de la frecuencia es directamente proporcional a los cambios en la potencia reactiva y puede ser escrita en términos de la potencia activa $\left(\mathrm{P}_{\mathrm{DG}}\right)$, el factor de calidad $\left(\mathrm{Q}_{\mathrm{f}}\right)$ y la frecuencia de resonancia $\left(f_{o}\right)$ :

$$
\Delta f=-\frac{f_{O}}{2 P_{D G} \cdot Q_{f}} Q
$$

La referencia de frecuencia en el control del inversor puede ser calculada según:

$$
f_{\text {ref }}=f_{g}+K_{f} \cdot\left(f-f_{g}\right)
$$

Donde $\mathrm{f}_{\mathrm{g}}$ es la frecuencia de la red; $\mathrm{f}$ es la frecuencia medida; $y \mathrm{~K}_{\mathrm{p}}$ es la constante que permite acelerar la detección de islanding $\mathrm{y}$, además, ser calculada teniendo en cuenta los parámetros de (9).

En la figura 4 se expone el diagrama de bloques del método basado en la variación simultánea de P y Q.

La desventaja que presenta éste método es que se pueden generar falsas detecciones de islanding

\section{FIGURA 4. Diagrama de bloques del método} de inyección de $P$ y $Q$.

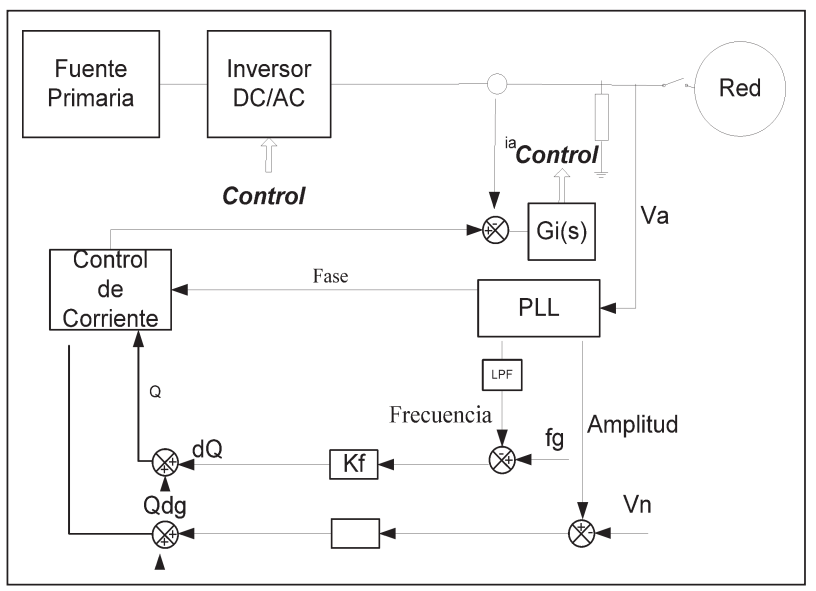

cuando se incorporan muchos inversores a la red. Así mismo, pueden aparecer problemas de inestabilidad por la inyección continua de perturbaciones por parte del inversor a la red.

\section{Métodos activos basados en inyección de armónicos}

Estos procedimientos se caracterizan por monitorear los cambios de la impedancia en la red debido a la inyección de un armónico o un subarmónico en particular (Zhang, et ál., 2006), (Ropp, M.; Begovic, M. y Rohatgi, A.; 1999), (Timbus, A. et ál, 2004). Además, asimilan que cuando la red está conectada, su impedancia es menor que la de la carga a la frecuencia armónica y, en consecuencia, la corriente armónica fluye hacia la red; pero cuando ésta se desconecta dicha corriente armónica fluye por la carga, lo que produce una tensión armónica que puede ser detectada. A continuación se explica el método basado en el PLL implementado por Ciobotaru, Agelidis y Teodorescu (2008).

Éste sistema se basa en la detección de los cambios de la impedancia de la red; al inyectar una corriente armónica (para éste caso de segundo orden) se modifica el ángulo de la corriente del inversor. Lo anterior se logra inyectando una señal sinusoidal sincronizada ciclo a ciclo, la cual obliga a modificar suavemente el ángulo de la corriente del inversor. En seguida, una señal realimentada es extraída 

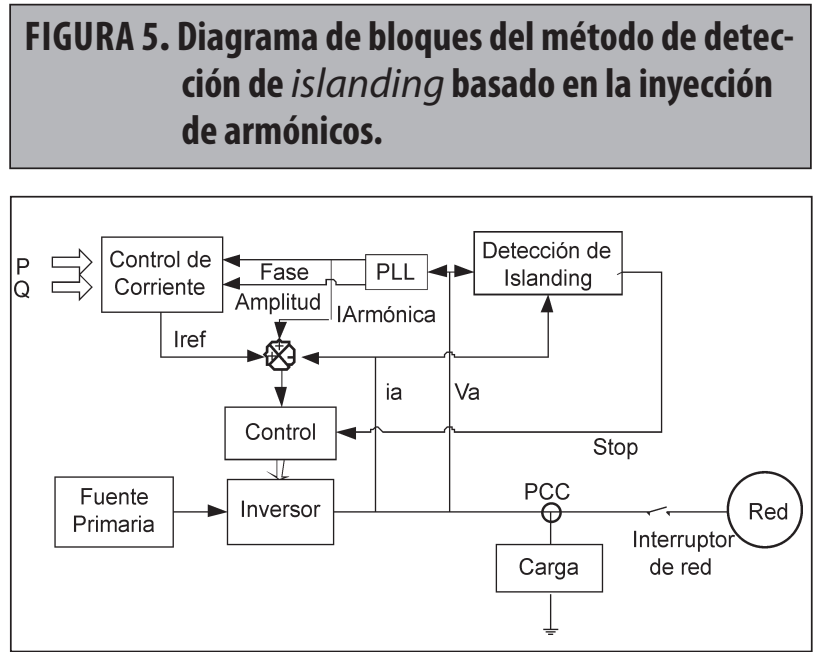

de la tensión en el PCC (componente $\mathrm{V}_{\mathrm{q}}$ ) como consecuencia de la señal armónica inyectada.

En la figura 5 se puede apreciar el esquema antiislanding basado en los cambios de la impedancia de red.

El principio de operación del método se describe a continuación; la señal inyectada $\mathrm{S}_{\text {inj }}$ se define como:

$$
S_{i n j}=k \cdot \sin \left(2 \cdot \theta_{P L L}\right)
$$

Donde k es la ganancia usada para escoger la cantidad de señal inyectada necesaria para la detección de islanding y $\theta_{\text {PLL }}$ es el ángulo aportado por el PLL. En (11) se observa que ha sido ingresado un segundo armónico. Éste será adicionado a la fase del PLL y posteriormente escalado por la magnitud de la corriente de referencia del sistema.

$$
i_{\text {ref }}=\operatorname{Iref} \cdot\left|\cos \left(\theta_{P L L}\right)+k \cdot \sin \left(2 \cdot \theta_{P L L}\right)\right|
$$

El valor de k se debe escoger teniendo en cuentas tres premisas: a) que sea lo suficientemente grande para poder detectar la condición de islanding; $b$ ) que su valor no sea tan grande como para producir un aumento del THD en una corriente superior a los límites establecidos por las normas; y c) que no afecte significativamente la amplitud de la referencia de corriente. En (12) la perturbación se estipula en términos de un porcentaje de la corriente de referencia — para dicho caso se empleó una perturbación del 1\% de amplitud, la cual cumple dichas condiciones.

Cuando el inversor se encuentra conectado a la red, la acción de agregar la perturbación descrita anteriormente se manifiesta en la señal realimentada de la tensión en el PCC. Ésta posee el doble de frecuencia en comparación con la fundamental y su amplitud se relaciona con el valor de la impedancia de red (dicha señal realimentada puede ser extraída de la Transformada de Park en términos de $\mathrm{V}_{\mathrm{q}}$ ). Después de la transformación, la frecuencia de la señal realimentada llega a ser la mitad, como se demuestra a continuación:

$$
v_{q}=-\sin (\theta) \cdot v_{\alpha}+\cos (\theta) \cdot v_{\beta}
$$

Considerando la tensión de realimentación $\left(\mathrm{V}_{\mathrm{a}}\right.$ y $V_{\beta}$ ) como un sistema de tensiones ortogonales debidas a la señal inyectada y normalizando las tensiones a y $\beta$, las cuales se pueden representar como:

$$
v_{\alpha}=\cos (2 \theta) y v_{\beta}=\cos \left(2 \theta-\frac{\pi}{2}\right)=\sin (2 \theta)
$$

Entonces, se obtiene:

$$
\begin{gathered}
v_{q}=-\sin (\theta) \cdot \cos (2 \theta)+\cos (\theta) \cdot \sin (2 \theta) \\
v_{q}=\sin (\theta)
\end{gathered}
$$

La señal de tensión realimentada puede ser extraída después de la Transformación de Park a través de un filtro resonante sintonizado a la frecuencia de la componente fundamental; posteriormente se detectan los picos en valor absoluto de la señal de $\mathrm{V}_{\mathrm{q}}$ y se calcula la media a $50 \mathrm{~Hz}$ y $5 \mathrm{~Hz}$. Finalmente, se toma la diferencia entre ambas medias con el fin de determinar si se encuentra fuera o dentro de un valor umbral determinado. Si está por fuera, se activa un retardo con el fin de establecer si dicha condición persiste y, de ser así, se activa la condición de islanding.

REVISTA CIENTÍFICA / ENERO -DICIEMBRE DE 2010 / No. 12 / BOGOTÁ, D.C. 
Las principales ventajas que ofrece éste método es la no alteración de los cruces por cero de la corriente, la posibilidad de inyectar — en todos los tiempos - la amplitud de la perturbación sin afectar significativamente el THD de corriente y, además, presenta una muy baja NDZ. No obstante, al tener una perturbación constante, degrada ligeramente la calidad de potencia y puede presentar inconvenientes para detectar el islanding ante ciertas perturbaciones de la red; podrían coincidir con el armónico inyectado.

\section{Propuesta de un nuevo método basado en la detección de perturbaciones en el PLL}

Se fundamenta en la adición de una corriente armónica a la referencia de corriente del inversor. La perturbación es generada por la modificación de la señal de fase del PLL, de modo que el ángulo que se introduce en la referencia de corriente del inversor $\left(\theta_{\mathrm{INV}}\right)$ —varía según (17). El esquema de la perturbación en el PLL se muestra en la figura 6 (Ciobotaru, M. et ál; 2010).

$$
\theta_{\mathrm{INV}}=\theta_{\mathrm{FPLL}}+\mathrm{k} \cdot \cos \left(\theta_{\mathrm{FPLL}}\right)
$$

Siendo k el porcentaje de perturbación introducido en el sistema:

\section{FIGURA 6. Perturbación en el PLL.}

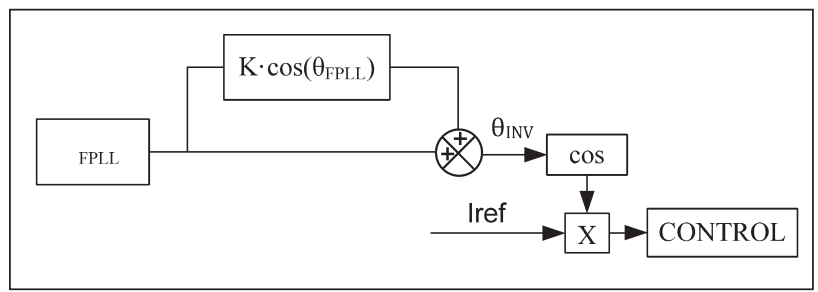

La figura 7 muestra la forma de onda de $\theta_{\text {INV }}$ para $\mathrm{k}=0.1$. El efecto de (17) es modificar la forma de onda de la corriente de salida del inversor, introduciendo un segundo armónico, como se muestra a continuación:

La forma de onda de la señal inyectada está definida por (18):

$$
\sigma_{i n j}=k \cdot \cos \left(\theta_{F P L L}\right)
$$
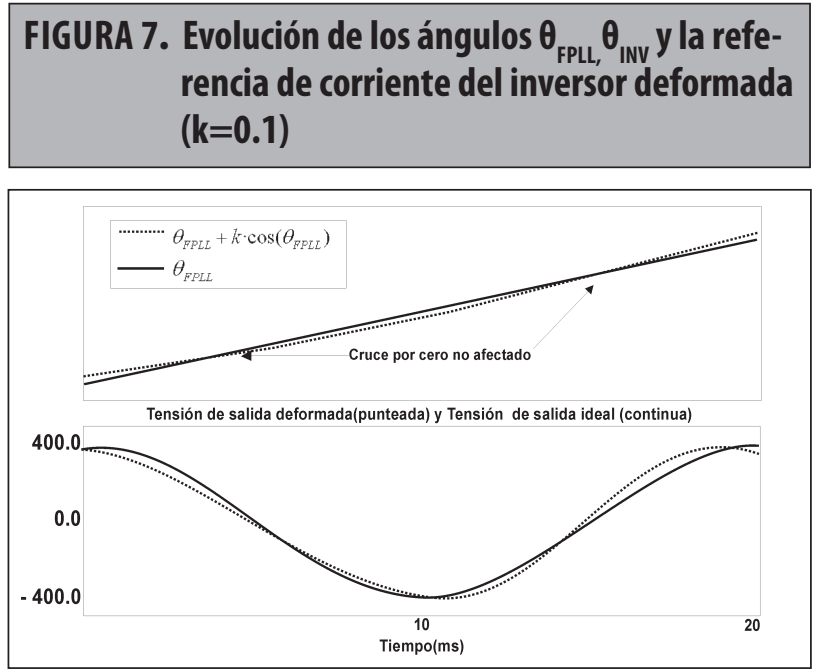

La referencia de fase de la corriente del inversor está dada por (19)

$$
\cos \theta_{I N V}=\cos \left(\theta_{F P L L}+\sigma_{i n j}\right)=\cos \left(\theta_{F P L L}+k \cdot \cos \left(\theta_{F P L L}\right)\right)
$$

Aplicando la relación trigonométrica (20) a (19), resulta (21):

$$
\begin{gathered}
\cos (A+B)=\cos (A) \cdot \cos (B)-\sin (A) \cdot \sin (B) \\
\cos \left(\theta_{F P L L}+k \cdot \cos \left(\theta_{F P L L}\right)\right)= \\
\cos \left(\theta_{F P L L}\right) \cdot \cos \left(k \cdot \cos \left(\theta_{F P L L}\right)\right)-\sin \left(\theta_{F P L L}\right) \cdot \sin \left(k \cdot \cos \left(\theta_{F P L L}\right)\right)
\end{gathered}
$$

Asumiendo que $\mathrm{k}<<1$, resulta la aproximación dada en (22) y, aplicándola a (21), se obtiene (23):

$$
\begin{gathered}
\sin \left(k \cdot \cos \left(\theta_{F P L L}\right)\right) \cong k \cdot \cos \left(\theta_{F P L L}\right), \text { para } k \ll 1 \\
\cos \left(k \cdot \cos \left(\theta_{F P L L}\right)\right) \cong 1, \text { para } k \ll 1 \\
\cos \left(\theta_{\mathrm{INV}}\right) \cong \cos \left(\theta_{\mathrm{FPLL}}\right)-\mathrm{k} \cdot \sin \left(\theta_{\mathrm{FPLL}}\right) \cdot \cos \left(\theta_{\mathrm{FPLL}}\right)
\end{gathered}
$$

Tomando en cuenta (24); (23) se puede reescribir como (25):

$$
\begin{gathered}
\sin (2 \cdot \theta)=2 \cdot \sin (\theta) \cdot \cos (\theta) \\
\cos \left(\theta_{I N V}\right) \cong \cos \left(\theta_{F P L L}\right)-\frac{k}{2} \cdot \sin \left(2 \cdot \theta_{F P L L}\right)
\end{gathered}
$$

Como se demuestra en (25), la adición del término $\mathrm{k} \cdot \cos \left(\theta_{\mathrm{FPLL}}\right)$ a $\theta_{\mathrm{FPLL}}$-al igual que en (19) - para pequeños valores de $\mathrm{k}(\mathrm{k}<<1)$ resulta en un segundo armónico en la señal de referencia de corriente del inversor. 
En consecuencia, una pequeña distorsión en la corriente de referencia del inversor es esperada (figura 7).

Así mismo, la forma de onda de la referencia de corriente del inversor no se ve afectada por los cruces por cero, mientras que los valores pico son desplazados en tiempo pero no modifican su valor.

Cuando la red está conectada, la tensión en el PCC es fijada por la red y no se ve alterada en su forma de onda. Sin embargo, cuando la red no está presente (situación de islanding), la tensión en el PCC seguirá la forma de onda de la corriente inyectada por el inversor. El método de detección de islanding propuesto se basa en medir la deformación de la forma de onda de la tensión en el PCC. Ésta deformación puede ser cuantificada por el cálculo de la diferencia de tiempo entre los picos de la tensión de la forma de onda en el PCC y los ideales. A pesar de la presencia de los armónicos impares en la tensión de red, los picos de tensión en el PCC con la red conectada se encontrarán centrados entre cada cruce por cero.

En la figura 8 se puede observar cómo la forma de onda de tensión ha sido deformada con la inclusión de un segundo armónico de pequeña amplitud $(\mathrm{k}=0.1)$.

\section{FIGURA 8. Medición de la deformación de la onda de tensión con $\mathrm{k}=0.1$}

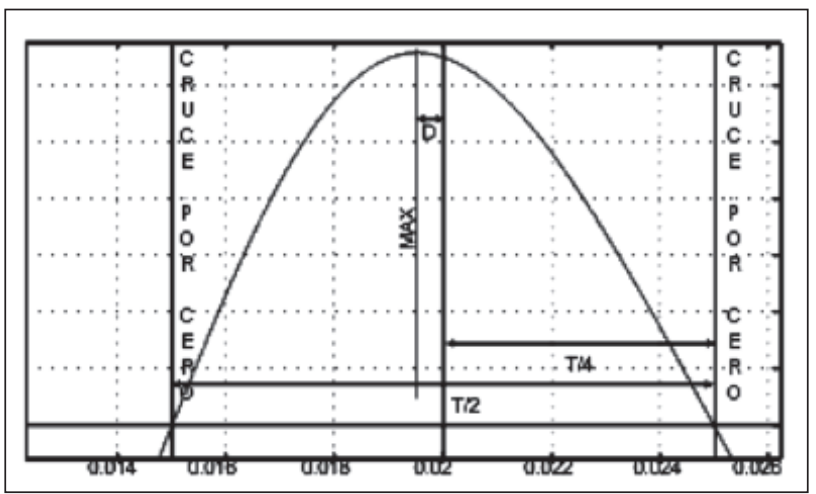

En la figura anterior se observa la manera en la que el pico se desplaza con respecto a una sinusoidal ideal. La diferencia de tiempo entre el valor central de los cruces por cero y el tiempo en que el pico real de la tensión ocurre, puede ser medida. Si ésta diferencia de tiempo es más alta que un valor umbral establecido, la condición de islanding es detectada. La señal de salida del PLL es la fase de la tensión a ser sincronizada y se encuentra disponible en el algoritmo de control.

En el sistema de control, la referencia de corriente es multiplicada por el coseno de la fase del PLL, así que los cruces por cero corresponden a $\theta_{\text {FPLL }}=\pi / 2$ y $3 \cdot \pi / 2$; mientras que los picos de tensión -positivos y negativos- con la red conectada son iguales a $2 \pi$ y $\pi$, respectivamente. Para la detección de los valores picos de la tensión en el PCC desde la fase del $\operatorname{PLL}\left(\theta_{\mathrm{FPLL}}\right)$, los instantes de tiempo cuando el $\theta_{\mathrm{FPLL}}=\pi$ y $2 \cdot \pi$ son medidos y restados de $\mathrm{T} / 2$ y $\mathrm{T}$ (siendo $\mathrm{T}$ el periodo de la tensión en el PCC).

El valor absoluto de la diferencia de tiempo calculado en cada medio ciclo se denomina D y el resultado es filtrado a través de un pasabajos $(10 \mathrm{~Hz})$, obteniendo, de ésta forma, un valor promedio (MV) que da una idea de la deformación de tensión en el PCC cuando ocurre el islanding.

Por su parte, un umbral de comparación es establecido para la variable promediada MV; determina en qué momento el sistema está o no en situación de islanding. Dicho umbral se encuentra definido como un porcentaje de desviación y es transformado, posteriormente, en un valor de tiempo.

La diferencia de tiempo (D) entre los picos de la señal ideal y la señal distorsionada de la tensión en el PCC, puede ser calculada como:

$$
\begin{gathered}
V_{M A X} \rightarrow \cos \left(\theta_{F P L L}+k \cdot \cos \theta_{F P L L}\right)=1 \\
\theta_{F P L L}+k \cdot \cos \theta_{F P L L}=0 \\
\theta_{F P L L}=t(m s) \cdot 2 \cdot \pi / T(m s) \\
t(m s) \cdot 2 \cdot \pi / T(m s)=-k \cdot \cos (t(m s) \cdot 2 \cdot \pi / T(m s))
\end{gathered}
$$

Para $\mathrm{f}=50 \mathrm{~Hz}$ y $\mathrm{k}=0.05$, se tiene:

$$
t(m s)=-3.183 \cdot k(m s)
$$


El valor de tiempo obtenido en (30) puede ser expresado como un porcentaje del tiempo correspondiente a una cuarta parte del periodo de la red, llamado D\% (31)

$$
D \%=k \cdot(3.183 / 5) \cdot 100=k \cdot 10.98
$$

Por lo que para una $\mathrm{k}=0.05$ se tendrá una $\mathrm{D} \%=0.549 \%$

La desviación existente entre la corriente de referencia del inversor y el pico de la tensión en el PCC es de $0.5 \%$ en tiempo; por lo tanto, puede fijarse un umbral menor con el fin de detectar la desviación en el caso de islanding.

Por otra parte, un retardo es programado para verificar la situación de islanding y así prevenir falsas detecciones en presencia de transitorios o ruidos en las mediciones. Cuando el valor medio (MV) excede los umbrales, el retardo es mantenido por un periodo de $100 \mathrm{~ms}$ (correspondiente a 5 ciclos de red) antes de la desconexión del PCC. Lo anterior con fin de garantizar que la situación de islanding realmente se haya producido -éste retardo no excede lo establecido por el Estándar IEEE 9292000.

Las utilidades más destacables de éste método son: la medida de la magnitud no depende de los transitorios, su algoritmo presenta gran simplicidad, no afecta al MPPT, inyecta perturbaciones insignificantes en estado estable (no afecta a la estabilidad de red), no presenta NDZ, obtiene resultados similares con diferentes tipos de carga (R, RLC, no lineal) y sus tiempos de detección son cortos (menores a 200ms).

Pueden señalarse dos desventajas del método propuesto: $a)$ una pequeña corriente, correspondiente a un segundo armónico, es inyectada a la red por el inversor y b) excesivos armónicos de tensión en el PCC pueden causar falsas detecciones. Además, exhibe problemas cuando se presenta una distorsión de 5to armónico en la tensión del PCC superior al $5.5 \%$-se encuentra por encima de lo establecido por los estándares internacionales (5\%).

\section{RESULTADOS DE LA SIMULACIÓN}

En esta sección se exponen los principales resultados de las simulaciones según los métodos expuestos anteriormente. Dichas prácticas se realizaron en PSIM $^{\mathrm{TM}}$ y fueron probadas sobre el microinversor presentado en el sección 2 -siguiendo los requerimientos del Estándar IEEE 929-2000 en cuanto al tipo de carga y factor de calidad de la misma se refiere. En todos los casos se utilizó una carga RLC paralelo $(\mathrm{R}=120 \Omega, \mathrm{L}=153 \mathrm{mH}, \mathrm{C}=66 \mathrm{uF})$ con un factor de calidad de 2.5

En la figura 9(a) se muestran los resultados del método de detección de islanding basado en la variación de la potencia activa mediante el uso de la
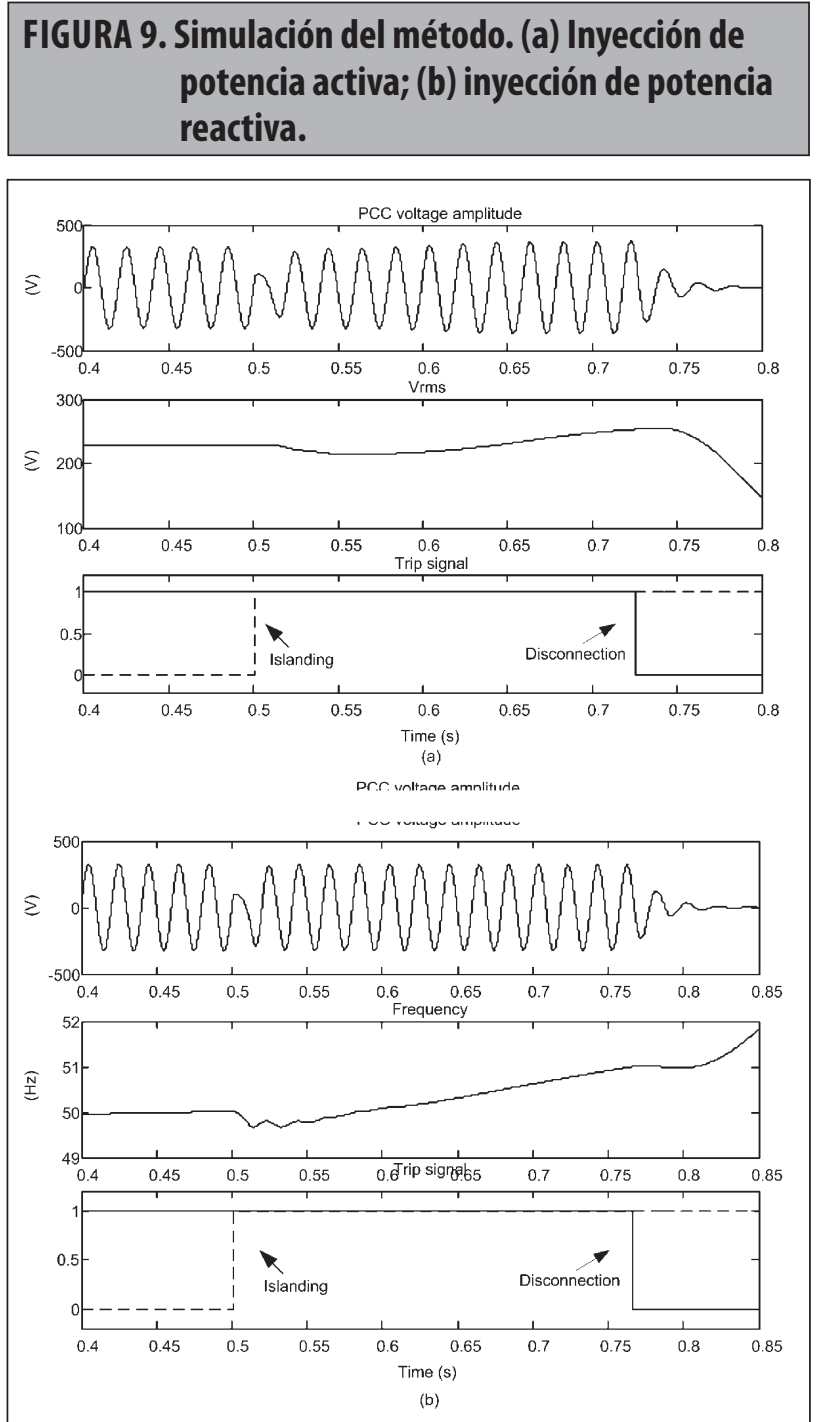

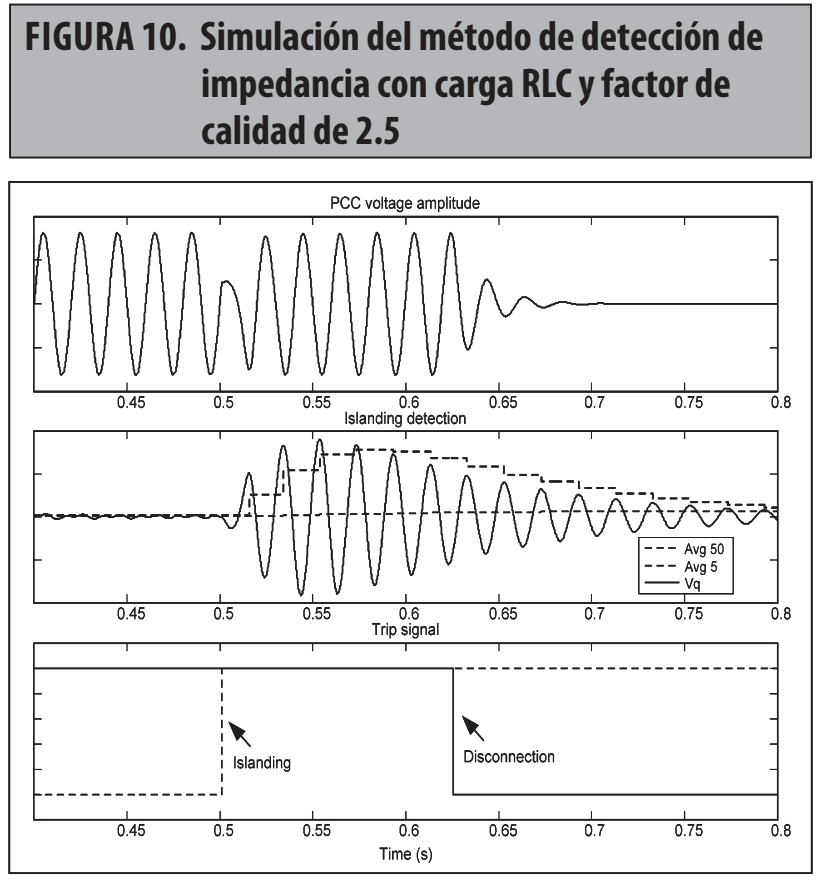

realimentación de tensión. En la gráfica se puede observar cómo se produce la desconexión del microinversor dentro de los tiempos establecidos por los estándares (IEEE Std. 929; 2000). Por su parte, en la figura 9(b) se obtienen resultados similares a los conseguidos anteriormente para el método de detección de islanding basado en la variación de la potencia reactiva (a través del uso de la realimentación de frecuencia). El valor de las constantes $\mathrm{K}_{\mathrm{v}} \mathrm{y}$ $\mathrm{K}_{\mathrm{f}}$ determinan el tiempo de respuesta del método.

En la figura 10 puede apreciarse la forma en la que, al producirse la situación de islanding, la componente $\mathrm{V}_{\mathrm{q}}$ cambia como resultado de la variación de impedancia a la frecuencia del armónico inyectado; lo que permite detectar la condición de islanding.

En la figura 11 se aprecian los resultados de simulación para el método propuesto (basado en la detección de perturbaciones en el PLL). En la gráfica superior se presenta la evolución de la tensión en el PCC. Entre tanto, la gráfica central muestra el tiempo en el cual ocurre el islanding y el tiempo en el cual el método lo detecta. La gráfica inferior representa la evolución de la variable medida MV. En la figura es evidente que el tiempo de desconexión del inversor cumple con los valores establecidos por los estándares.
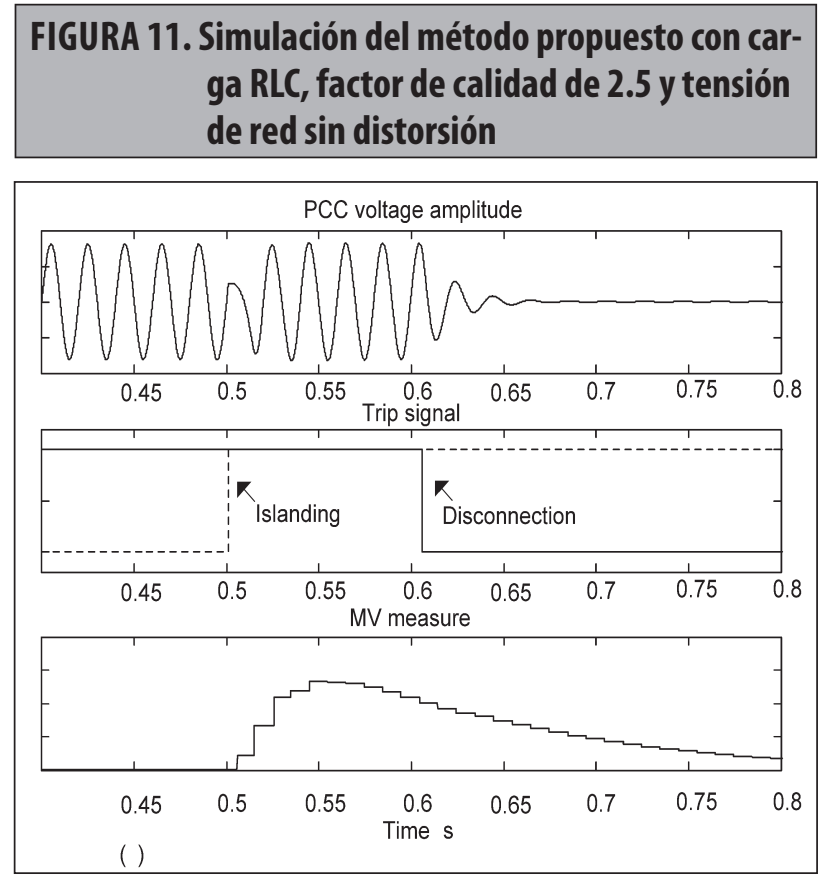

Para todos los métodos simulados anteriormente el THD en corriente nunca superó el 3.2\%, siendo el caso más crítico en los métodos de variación de potencia activa y potencia reactiva. Mientras tanto, para los otros métodos, el $\mathrm{THD}_{\mathrm{i}}$ no aumentó de forma significativa ya que la perturbación sólo se aplicó bajo condiciones determinadas y su magnitud se hizo tan pequeña como los métodos lo permiten. En la tabla 1 se presenta un cuadro comparativo de los tiempos de detección de los diferentes métodos.

\section{TABLA 1. Comparación de los diferentes métodos activos para detección de islanding.}

\begin{tabular}{|l|l|}
\hline Método & Tiempo de detección \\
\hline Variación de la potencia activa & $0.22 \mathrm{~s}$ con $\mathrm{Kv}=10$ \\
\hline Variación de la potencia reactiva & $0.26 \mathrm{~s}$ con $\mathrm{K}_{\mathrm{f}}=3$ \\
\hline Detección de impedancia & $\begin{array}{l}0.1 \mathrm{a} 0.2 \mathrm{~s} \text { en función del tamaño } \\
\text { de la perturbación. }\end{array}$ \\
\hline Método propuesto & $\begin{array}{l}\text { Ajustable de acuerdo al retardo } \\
\text { programado (para este caso 0.1s). }\end{array}$ \\
\hline
\end{tabular}

\section{CONCLUSIONES}

En este artículo se describieron las diferentes técnicas para detección de islanding (pasivas y activas) con fin de encontrar un mecanismo adecuado

REVISTA CIENTÍFICA / ENERO -DICIEMBRE DE 2010 / No. 12 / BOGOTÁ, D.C. 
para identificar el momento en que la red eléctrica es desconectada. Así mismo, fueron simuladas diferentes técnicas activas residentes en el inversor y se propuso una nueva técnica activa basada en el cambio de la forma de onda de la tensión de salida por inyección de un armónico de segundo orden. Finalmente, se realizaron simulaciones de los diferentes métodos activos y se diseñó una tabla comparativa en términos de tiempo de actuación del método y THD de corriente, con lo cual se estableció que los que presentaban un mejor comportamiento eran el basado en medición de impedancia y el método propuesto.

\section{REFERENCIAS}

- IEAPVPS T5-09. (2002), "Evaluation of islanding detection methods for photovoltaic utility interactive power systems", Reporte Técnico del International Energy Agency Implementing agreement on Photovoltaic Power Systems, USA

- Mahat, P.; Chen, Z. y Bak-Jensen, B., (2008, abril), "Review of islanding detection methods for distributed generation", en Electric Utility Deregulation and Restructuring and Power Technologies, vol. 6, núm., pp. 2743-2748.

— De Mango, F. et ál. (2006), "Overview of AntiIslanding Algorithms for PV Systems. Part I: Passive Methods", en Proc. of the 12th International Conference on Power Electronics and Motion Control, pp.1878-1883.

— Liserre, M. et ál. (2004), "Power converters and control of renewable energy systems", en Proc. of ICPE, pp. 1-19.

— Liang, J. et ál. (2003), "Hybrid control of multiple inverters in an island-mode distribution system," en IEEE 34th annual conference on power electronics specialist, vol. 1, pp.61-66.

- Ciobotaru, M.; Teodorescu, R. y Blaabjerg, F. (2006), "A new single phase PLL structure ba- sed on second order generalized integrator", en Record of IEEE PESC, pp. 1511-1516.

— Liserre, M. et ál. (2005), "Synchronization methods for three phase distributed power generation systems. An overview and evaluation," en PESC.

—Esram. T y Chapman, P. (2007, junio), “Comparison of Photovoltaic Array Maximum Power Point Tracking Techniques," en Energy Conversion, IEEE Transactions, vol. 22, núm. 2, pp.439-449.

- Kazmierkowski, M.; Krishnan, R. y Blaabjerg, F. (2002), Control in power electronics. Londres, London Academic Press.

- IEEE recommended practice for grid interface of photovoltaic (PV) systems, IEEE Std. 929, 2000.

— Jeraputra, C. y Enjeti, P. (2004), "Development of a robust anti-islanding algorithm for grid interconnection of distributed fuel cell powered generation" en IEEE Transactions on Power Electronics, vol.19, núm. 5, pp. 1163 -1170.

— Huili, S.; Lopes, L. y Zhixiang, L. (2004) "Analysis and comparison of islanding detection methods using a new load parameter space", en Proc. of the 30th Annual Conference of the IEEE Industrial Electronics Society, vol. 2, pp. $1172-1177$.

- Ye, Z. et ál. (2004), "A new family of active antiislanding schemes based on DQ implementation for grid-connected inverters," en Proc. of the 35th Annual IEEE Power Electronics Specialists Conference, pp. 235-241.

— Lopes, L. y Huili, S. (2006), "Performance assessment of active frequency drifting islanding detection methods", en IEEE Trans. on Energy Conversion, vol. 21, núm. 1, pp. 171-180.

— Wang, X et ál. (2007), "Impact of DG Interface Controls on the Sandia Frequency Shift An- 
tiislanding Method", en IEEE Trans. on Energy Conversion, vol. 22, núm. 3, pp. 792-794.

—Zhang, C. et ál. (2006) "A Novel Active Islanding Detection Method of Grid-connected Photovoltaic Inverters Based on CurrentDisturbing", en Power Electronics and Motion Control Conference, vol.3, pp.1-4.

- Ropp, M.; Begovic M. y Rohatgi, A. (1999), "Prevention of islanding in grid-connected photovoltaic systems", en Progress in Photovoltaics: Research and Applications [en línea], disponible en http://www3.interscience.wiley. com/cgi-bin/fulltext/40007275/PDFSTART, recuperado: 16 de enero de 2010.
— Timbus, A. et ál. (2004, septiembre), "Online grid measurement and ENS detection for PV inverter running on highly inductive grid", en IEEE Power Electronics Letters, pp. 77-82.

- Ciobotaru, M.; Agelidis V. y Teodorescu. R. (2008, junio), "Accurate and less-disturbing active anti-islanding method based on PLL for grid-connected PV Inverters", en Power Electronics Specialists Conference, pp.4569-4576.

—Ciobotaru, M. et ál. (2010, junio), "Accurate and Less-Disturbing Active Antiislanding Method Based on PLL for Grid-Connected Converters," en IEEE Transactions on Power Electronics, pp.1576-1584. 